\title{
Commentary: The intercoronary pillar-Not necessarily an innocent bystander
}

\author{
Luis E. Martínez-Bravo, MD, ${ }^{\mathrm{a}, \mathrm{b}}$ and Carlos M. Mery, MD, MPH ${ }^{\mathrm{b}}$
}

\footnotetext{
From the ${ }^{\mathrm{a} E s c u e l a}$ de Medicina, Tecnológico de Monterrey, Monterrey, Mexico; and ${ }^{\mathrm{b}}$ Texas Center for Pediatric and Congenital Heart Disease, Department of Surgery and Perioperative Care, University of Texas Dell Medical School, Dell Children's Medical Center, Austin, Tex.

Disclosures: Authors have nothing to disclose with regard to commercial support.

Received for publication Feb 10, 2019; accepted for publication Feb 11, 2019; available ahead of print April 5, 2019.

Address for reprints: Carlos M. Mery, MD, MPH, Texas Center for Pediatric and Congenital Heart Disease, University of Texas Dell Medical School, Dell Children's Medical Center, 4900 Mueller Blvd, Suite 3S.003, Austin, TX 78723 (E-mail: cmery@austin.utexas.edu).

J Thorac Cardiovasc Surg 2019;158:218-9

$0022-5223 / \$ 36.00$

Copyright (c) 2019 by The American Association for Thoracic Surgery

https://doi.org/10.1016/j.jtcvs.2019.02.037
}

Unroofing of an intramural segment is the most common surgical procedure performed for anomalous aortic origin of a coronary artery (AAOCA). ${ }^{1}$ The incidence of aortic insufficiency (AI) after unroofing seems to be underappreciated. Some studies have shown that AI occurs in as many as $17 \%$ of patients and may develop or progress with time. ${ }^{2,3}$

In this issue of the Journal, Yerebakan and colleagues ${ }^{4}$ describe the results of universal resuspension of the intercoronary commissure regardless of whether the commissure is taken down. They hypothesize that unroofing above the commissure alters the support of the valve by leading to a drop of the commissural level equal to half the diameter of the unroofed coronary artery. They compared 9 patients who did not have commissural resuspension with 17 who did. One patient who did not have commissural resuspension had progressive $\mathrm{AI}$, required aortic valve replacement 7 years later, and died of aortic valve endocarditis 11 years after unroofing. At last follow-up, which was variable between the groups, all patients who had commissural resuspension had no or trivial AI, whereas 6 of the 9 patients who did not have commissural resuspension had mild or greater AI.

This study pertinently suggests that the integrity of the intercoronary commissure, although necessary, is not sufficient to support the aortic valve adequately. Support of the valve is dependent on cranial traction and on support of the commissure itself. If one pays close attention to the aortocoronary complex, there is a segment of the aortic wall just cranial to the intercoronary commissure that is thicker than the rest of the wall. This segment, which we have called the intercoronary pillar and can be quite thick in some patients (Figure 1), not only seems to be important for valve support but also may play a significant role in the pathophysiology of AAOCA. The coronary artery arising from the incorrect sinus usually

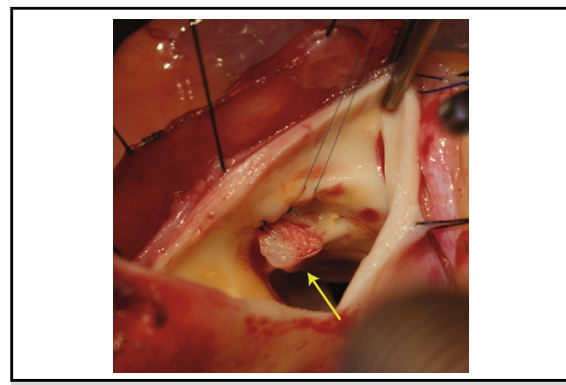

The intercoronary pillar can be quite thick in some patients.

\section{Central Message}

The intercoronary pillar, a thickening of the aortic wall above the commissure, may contribute to both aortic valve support and the pathophysiology of anomalous aortic origin of a coronary artery.

See Article page 208.

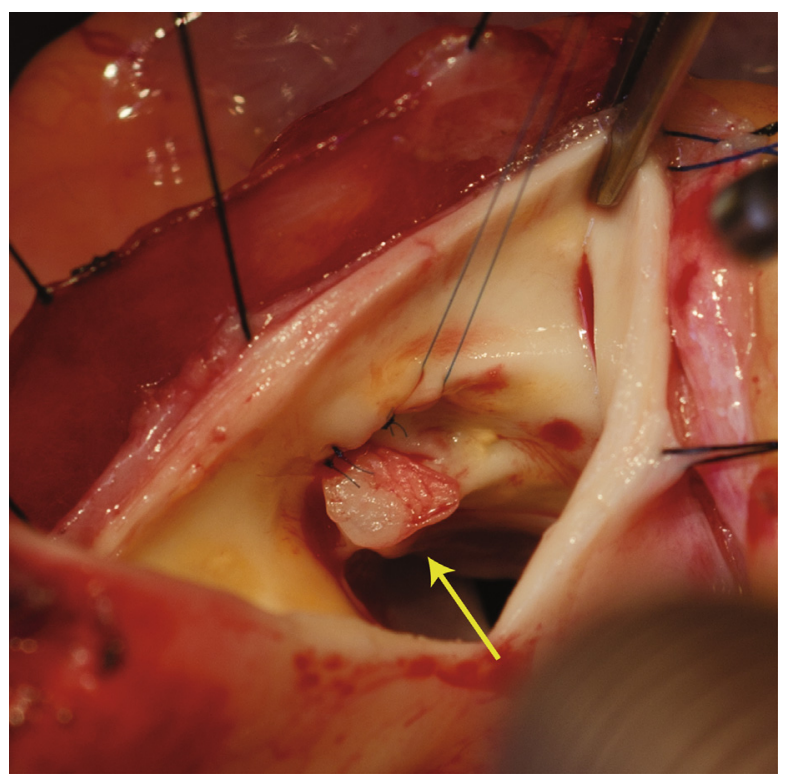

FIGURE 1. The intercoronary pillar, a thickening of the aortic wall extending from the intercoronary commissure cranially toward the sinotubular junction, can be quite thick in some patients (arrow). This pillar likely contributes to the aortic valve support and may play a role in the pathophysiology of anomalous aortic origin of a coronary artery. (@2014 Texas Children's Hospital, reprinted with permission.) 

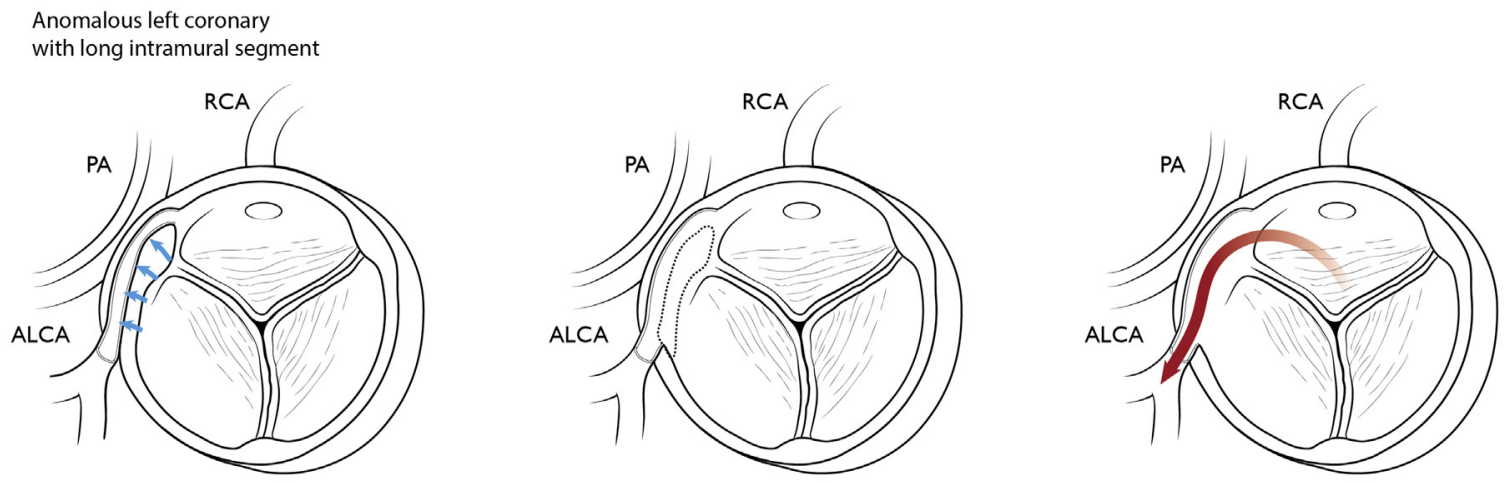

A

Anomalous left coronary

with short intramural segment

B
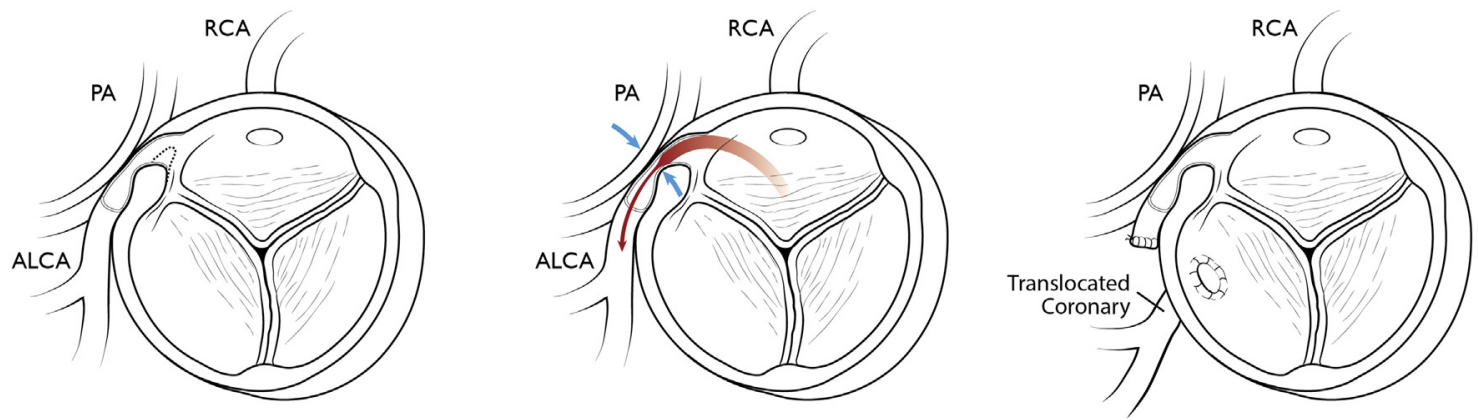

FIGURE 2. Effect of surgical unroofing of an anomalous coronary artery. A, Unroofing of a long intramural segment increases the size of the ostium, eliminates the intramural portion of the anomalous coronary, and places the ostium in the correct sinus, away from the intercoronary pillar. B, Unroofing of a short intramural segment increases the size of the ostium and eliminates the intramural portion of the coronary artery but may leave the coronary artery in close proximity to the intercoronary pillar and at risk for compression. An alternative surgical intervention, such as coronary translocation, may be preferred. $R C A$, Right coronary artery; PA, pulmonary artery; ALCA, anomalous left coronary artery. (@2016 Texas Children’s Hospital, reprinted with permission.)

travels behind and in close proximity to this thickened segment. Unroofing of a long intramural segment will move the ostium into the correct sinus and away from the intercoronary pillar (Figure 2). In contrast, unroofing of a short intramural segment may leave the coronary artery in close proximity to the pillar and potentially at risk for compression. In our experience, ${ }^{5}$ patients with unroofing of short intramural segments may show persistent compression of the coronary after unroofing. Alternative procedures, such as coronary translocation, may be preferable for these patients. This would become more important if we resuspend the commissure after unroofing a short intramural segment, ${ }^{4}$ because this would place the intercoronary pillar back closer to the ostium and potentially lead to persistent obstruction.

It is clear that despite the ability to achieve good perioperative outcomes, surgical intervention for AAOCA is not completely benign. It is incumbent on us to carefully weigh all the risks and benefits for a particular patient, especially given all the uncertainty surrounding the natural history of
AAOCA. In the meantime, careful consideration ought to be made to resuspending the commissure of these patients at the time of surgical unroofing.

\section{References}

1. Poynter JA, Bondarenko I, Austin EH, DeCampli WM, Jacobs JP, Ziemer G, et al Congenital Heart Surgeons' Society AAOCA Working Group. Repair of anomalous aortic origin of a coronary artery in 113 patients: a Congenital Heart Surgeons' Society report. World J Pediatr Congenit Heart Surg. 2014;5:507-14.

2. Nees SN, Flyer JN, Chelliah A, Dayton JD, Touchette L, Kalfa D, et al. Patient with anomalous aortic origin of the coronary artery remain at risk after surgical repair. J Thorac Cardiovasc Surg. 2018;155:2554-64.e3.

3. Fabozzo A, DiOrio M, Newburger JW, Powell AJ, Liu H, Fynn-Thompson F, et al. Anomalous aortic origin of coronary arteries: a single-center experience. Semin Thorac Cardiovasc Surg. 2016;28:791-800.

4. Yerebakan C, Ozturk M, Mota L, Sinha L, Gordish-Dressman H, Jonas R, et al. Complete unroofing of the intramural coronary artery for anomalous aortic origin of a coronary artery: the role of commissural resuspension? J Thorac Cardiovasc Surg. 2019;158:208-17.e2.

5. Mery CM, De León LE, Molossi S, Sexson-Tejtel SK, Agrawal H, Krishnamurthy R, et al. Outcomes of surgical intervention for anomalous aortic origin of a coronary artery: a large contemporary prospective cohort study. $J$ Thorac Cardiovasc Surg. 2018;155:305-19.e4. 